\title{
Preparation and characterization of CdS nanocrystallites in nafion
}

\author{
P NANDAKUMAR, C VIJAYAN, Y V G S. MURTI*, \\ K DHANALAKSHMI ${ }^{\dagger}$ and G SUNDARARAJAN \\ Department of Physics, 'Department of Chemistry, Indian Institute of Technology, \\ Madras 600 036, India
}

\begin{abstract}
Perfluoroethylene sulfonic acid polymer (NAFION) films are subjected to ion exchange in the medium of aqueous solutions of cadmium acetate, followed by ammonia passivation. The films are then treated with hydrogen sulfide gas for prescribed times. X-ray powder diffraction data of these samples have been analyzed for estimating the sizes of the nanocrystallites. The optical absorption spectra of the samples show an absorption edge beginning at $525 \mathrm{~nm}$ for the largest size clusters. A broad absorption band appears with a maximum around $410 \mathrm{~nm}-\mathbf{4 4 0} \mathrm{nm}$ in the smaller size clusters.
\end{abstract}

Keywords. Quantum dots; semiconductors; CdS; nanocrystallites; nafion.

\section{Introduction}

Remarkable quantum confinement effects have been observed in semiconductor quantum dot materials such as CdS doped glasses prepared by melt quenching and sol-gel techniques (Ekimov and Efor 1988; Reisfeld and Minti 1994). Nanosize CdS crystallites in the ionic polymer nafion (perfluoroethylene sulphonic acid) have been previously reported (Wang et al 1990). The microcrystallites are prepared by ion exchange in cadmium acetate solution and subsequent treatment with hydrogen sulfide gas. The crystallite size in these structures depend on the initial $\mathrm{Cd}^{2+}$ ion concentration in the solution. The crystallite sizes can be varied by annealing the sample for different time durations (Wang et al 1990). It was reported that size characterization of the sample becomes difficult as the particle size decreases below $4 \mathrm{~nm}$. Here we report the preparation of CdS nanocrystallites of different sizes less than $2 \mathrm{~nm}$ in nafion film and their characterization through XRD and optical absorption.

\section{Experimental}

Nafion films of thickness $0.18 \mathrm{~mm}$ are cleaned in boiling $70 \%$ nitric acid for $30 \mathrm{~min}$ and then repeatedly washed in boiling water till the $\mathrm{pH}$ of the water bath is neutral. The film is then soaked in $0.5 \mathrm{M}$ aqueous cadmium acetate solution for time durations varying from 8 to $20 \mathrm{~h}$. The film is dried under vacuum for $1 \mathrm{~h}$ and exposed to ammonia gas for $30 \mathrm{~min}$ and again dried under vacuum for $1 \mathrm{~h}$. The film is then treated with hydrogen sulfide gas for 15 to 20 min followed by drying under vacuum to yield CdS nanocrystallites.

The XRD is taken by step scanning with a step width of $0.02^{\circ}$ and $10 \mathrm{sec}$ integrating time. A profile fitting program (PROFIT)(Toraya 1985) is used to deduce the full width at half maximum (W). The fitting takes into account the amorphous background, the presence of $K_{\alpha 1}, K_{\alpha 2} \mathrm{X}$-ray lines, asymmetry in the Bragg peaks and tail decay rates of

\footnotetext{
*Author for correspondence
} 
the peaks. The overall goodness of the fit is determined by the reliability index defined by

$$
R=\left\{\frac{\sum_{i=1}^{N} w_{i}\left[y\left(x_{i}\right)_{\mathrm{obs}}-y\left(x_{i}\right)_{\mathrm{calc}}\right]^{2}}{\sum_{i=1}^{N} w_{i} y\left(x_{i}\right)_{\mathrm{obs}}^{2}}\right\}^{1 / 2},
$$

where $y\left(x_{i}\right)_{\text {obs }}$ are the observed ordinates for the angle $x_{i}, y\left(x_{i}\right)_{\text {calc }}$ are the values calculated. $w_{i}$ are the weightages of the points taken in proportion to the relative heights. $N$ is the number of data points in the fit.

The best fit corresponding to the minimum value of $R$ is used. The crystallite size is determined by using the Scherrer's formula (Cullity 1978) for the mean diameter of the particle

$$
D=\frac{\lambda}{W \cos (\theta)}
$$

where $\theta$ is the Bragg angle, $\lambda$ the wavelength of the $\mathrm{X}$-rays. The optical absorption spectra of the samples are recorded by a Hitachi U3400 Recording Spectrophotometer.

\section{Results and discussion}

Four sets of samples were prepared by varying the duration of the ion exchange process. These are denoted by S1, S2, S3 and S4. Figure 1 shows the XRD pattern containing the Bragg diffraction peaks due to the cubic phase $\beta$-CdS as well as a diffuse wide maximum due to the amorphous polymer matrix. The profile calculated by the PROFIT is also shown (continuous curve). The input value of the amorphous peak

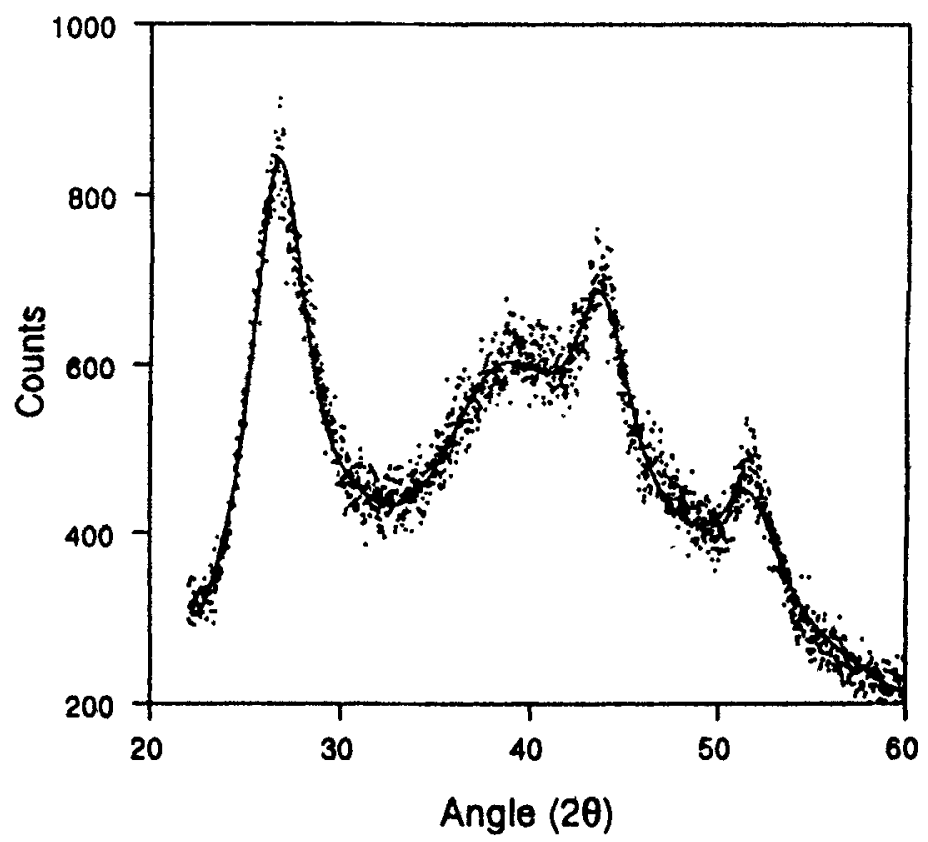

Figare 1. XRD of CdS in nafion. 


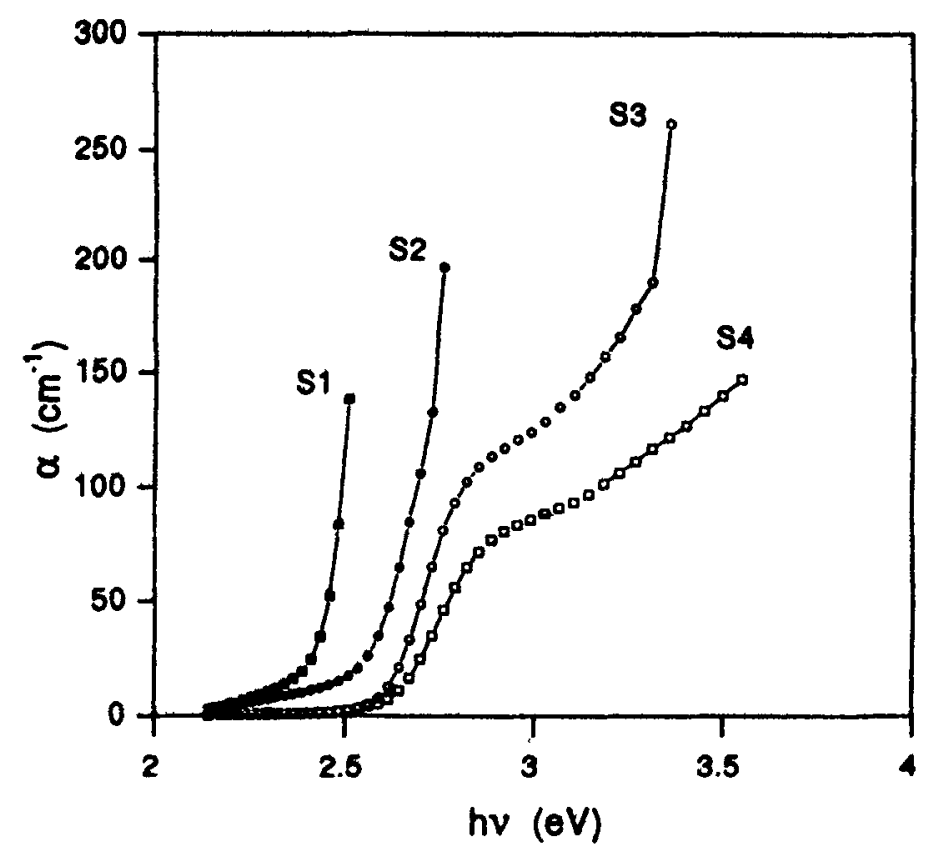

Figure 2. Optical absorption spectra of CdS in nafion.

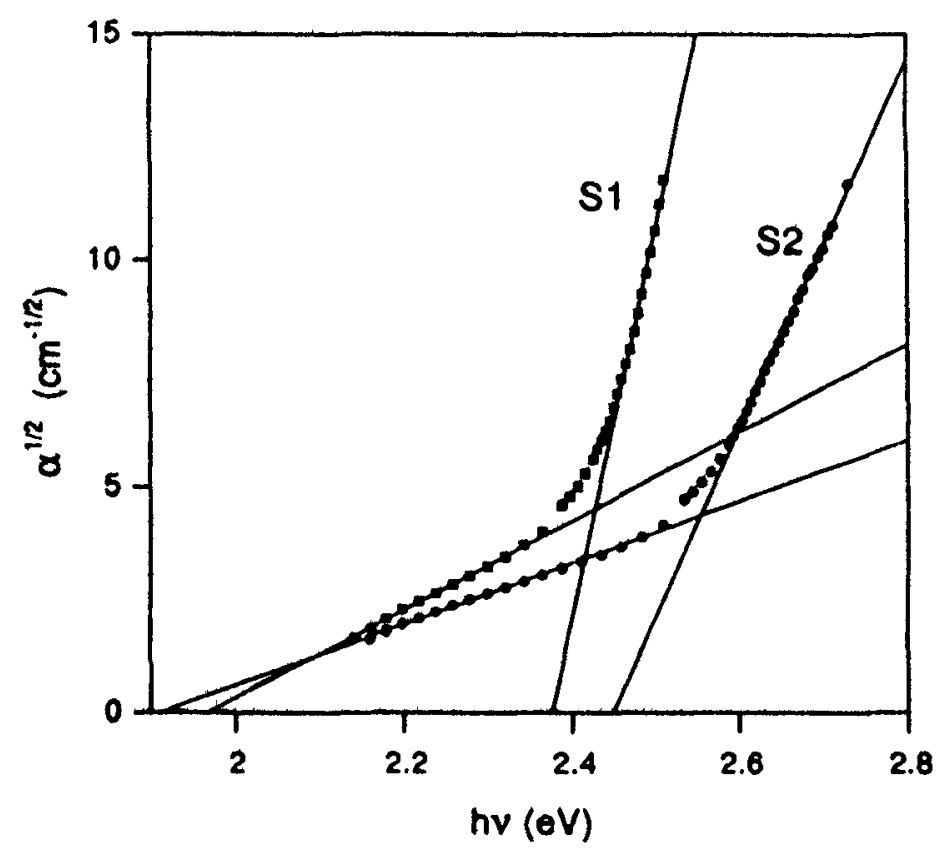

Fhare 3. Plot of $\alpha^{1 / 2}$ vs hv.

width is varied systematically to minimize $R$. Samples $S 1$, S2 (ion exchange duration $20 \mathrm{~h}$ and $14 \mathrm{~h}$ respectively) correspond to the mean diameters of $6 \mathrm{~nm}$ and $2 \mathrm{~nm}$ respectively. 
Figure 2 shows the optical absorption spectra of the four samples. Analyzing the tail and absorption edge in the case of $\mathbf{S 1}$ and $\mathbf{S} 2$ we find that it corresponds to an indirect allowed band to band transition (Smith 1961) (figure 3 ) with a band gap energy $\left(E_{\mathrm{g}}\right)$ $2 \cdot 17 \mathrm{eV}$ and $2 \cdot 18 \mathrm{eV}$ and phonon energy $0.20 \mathrm{eV}$ and $0.27 \mathrm{eV}$ for $\mathrm{S} 1$ and $\mathrm{S} 2$, respectively. The absorption spectra of the samples 3 and 4 show a broad absorption band with a maximum around $410 \mathrm{~nm}-440 \mathrm{~nm}$. These bands are presumably due to excitonic transitions as interpreted in an earlier work (Wang et al 1990).

\section{Acknowledgements}

We are thankful to Dr P Kesavan Nair for providing a copy of the PROFIT software and for help in implementing it. Some of the XRD pictures were taken at the Central Diffraction Unit, IIT Madras.

\section{References}

Cullity B D 1978 Elements of $X$-ray diffraction (Philippines: Addison-Wesley Pub. Co. Inc.)

Ekimov A I and Efor A L 1988 Phys. Status Solidi B150 627

Reisfeld R and Minti H 1994 J. Sol-Gel Sci. \& Technol. 2641

Smith R A 1961 Semiconductors (London: Cambridge University Press)

Toraya H 1985 J. Appl. Cryst. 18, 351

Wang Y, Suna A, Mcltugh J, Hinlinsky E F, Lucas P A and Johnson R D 1990 J. Chem. Phys. 926927 